\title{
Experimental Simulation of Ground Motion Effects
}

\author{
M.J. Syphers ${ }^{a *}$, M. Ball ${ }^{b}$, B. Brabson ${ }^{b}$, J. Budnick, D.D. Caussyn ${ }^{b}$, A.W. Chao ${ }^{a}$, J. Collins $^{b}$, V. Derenchuk ${ }^{b}$, \\ S. Dutt ${ }^{a}$, G. East ${ }^{b}$, M. Ellison ${ }^{b}$, T. Ellison ${ }^{b}$, D. Friesel ${ }^{b}$, W. Gabella ${ }^{d}$, B. Hamilton ${ }^{b}$ H. Huang ${ }^{b}$, \\ W.P. Jones ${ }^{b}$, S.Y. Lee ${ }^{b}$, D. Li $^{b}$, M.G. Minty ${ }^{c}$, S. Nagaitsev ${ }^{b}$, K.Y. Ng ${ }^{d}$, X. Pei ${ }^{b}$, G. Rondeau ${ }^{b}$,

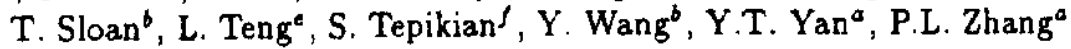

\section{Abstract}

Synchro-betatron coupling in a proton storage ring with electron cooling was studied by modulating a transverse dipole field close to the synchrotron frequency. The combination of the electron cooling and transverse field modulation on the synchratron oscillation is equivalent to a dissipative parametric resonant system. The proton bunch was observed to split longitudinally into two pieces, or beamlets, converging toward strange attractors of the dissipative system. These phenomena might be important to understanding the effect of ground vibration on the SSC beam, where the synchrotron frequency is about $4 \sim 7 \mathrm{~Hz}$, and the effect of power supply ripple on the RHIC beam, where the synchrotron frequency ramps through $60 \mathrm{~Hz}$ at $17 \mathrm{GeV} / \mathrm{c}$.

\section{INTRODUCTION}

The equation of motion for phase oscillations[1] of a particle in a synchrotron, in the absence of forced oscillations, is given by

$$
\ddot{\phi}+\omega_{2}^{2}\left(\sin \phi-\sin \phi_{0}\right)=0 \text {. }
$$

Here $\omega,=\omega_{0} \sqrt{\frac{h e V|\eta|}{2 \pi \beta^{2} E}}$ is the small amplitude synchrotron angular frequency at $\phi_{0}=0, \omega_{0}$ is the angular revolution frequency, and $h, V$, and $\phi_{0}$ are respectively the harmonic number, the peak. If voltage and the synchronous phase angle. The speed of the particle is $\beta c, E$ is the energy, and $\eta$ is the phase slip factor. For this experiment, we have $h=1, \eta \approx-0.86, \phi_{0}=0$ and $f_{0}=\frac{\omega_{0}}{2 \pi}=1.03168 \mathrm{MHz}$ at $45 \mathrm{MeV}$ proton kinetic energy. We chose an if voltage of $41 \mathrm{~V}$ to obtain a synchrotron frequency of $262 \mathrm{~Hz}$ in order to avoid odd harmonics of the $60 \mathrm{~Hz}$ ripple. The synchrotron tune is given by $\nu_{s y n}=\frac{\omega_{s}}{\omega_{0}}=2.54 \times 10^{-4}$.

Transversely, particles are executing betatron oscillations about the closed orbit of the accelrator. Horizontal and vertical tunes $\nu_{x}=3.828$, and $\nu_{z}=4.858$ were chosen for this experiment to avoid nonlinear betatron resonances. The corresponding fractional part of the horizontal betatron frequency was $\left(4-\nu_{x}\right) f_{0}=177 \mathrm{kHz}$.

- a SSC laboratory, 2550 Beckleymeade A venue, Dallas, TX 75237. 3946 (Operated by the Universities Research Association, Inc., for the U.S. Department of Energy under Contract No. DE-AC3589ER40486.); ${ }^{b} \mathrm{IUCF}$, Indians University, Bloomington, IN 47405; ${ }^{c}$ SLAC, MS26, Box 4349, Stanford, CA 94309; ¿ Fermilab, P.O. Box 500, Batavia, IL 60510; ' Argonne National Laboratory, APS, 9700 S. Cass A ve., IL60439; ' Brookhaven National Laboratory, Upton, NY 11973.

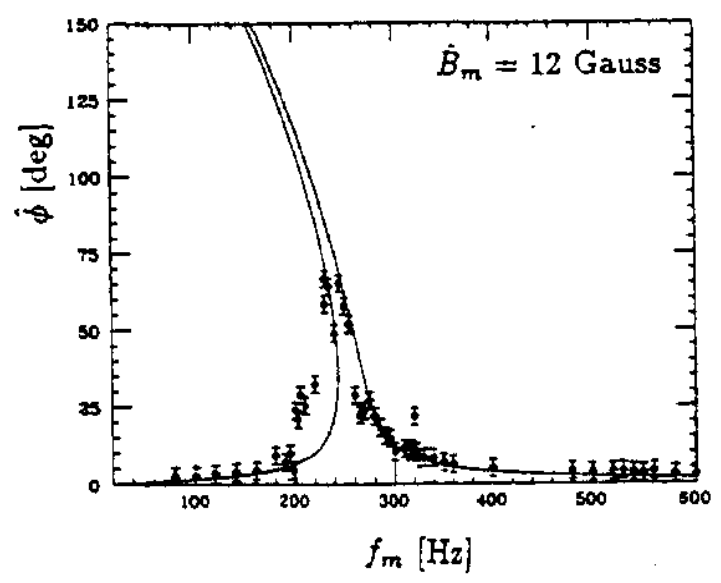

Figure 1. Phase amplitude response as a function of the modulation frequency near the synchrotron tune. Solid lines correspond to attractor solutions calculated with the parameter $a$ of $\mathrm{Eq} \cdot(3)$.

Coupled motion between the transverse and longitudinal degrees of freedom (synchro-betatron coupling) is important to electron storage rings, where the fractional parts of the synchrotron and betatron tunes are of the same order of magnitude. To the knowledge of the authors, synchrobetatron (SB) coupling has not been observed previously in proton storage rings, where the fractional part of the betatron and synchrotron tunes differ substantially.

The dominant effect of ground vibration or power supply ripple is that of a modulating dipole field. A slow adiabatic dipole modulation gives rise to a closed orbit modulation. Performance degradation can arise from nonadiabatic modulations, which occur naturally at resonance conditions, e.g. transverse modulation at the synchrotron frequency. In this paper, we present experimental data for transverse modulations producing SB coupling, similar to what may arise from ground vibrations or power supply ripple.

\section{THE EXPERIMENT}

The experimental procedure started with a single bunch of about $5 \times 10^{8}$ protons at at $45 \mathrm{MeV}$ kinetic energy. The cycle time was $11 \mathrm{~s}$. The injected beam was electron-cooled and was simultaneously modulated by a small dipole of effective length $\ell=0.27 \mathrm{~m}$. The horizontal dispersion function was $D_{x} \approx 4.0 \mathrm{~m}$ at this dipole's location. At the end 

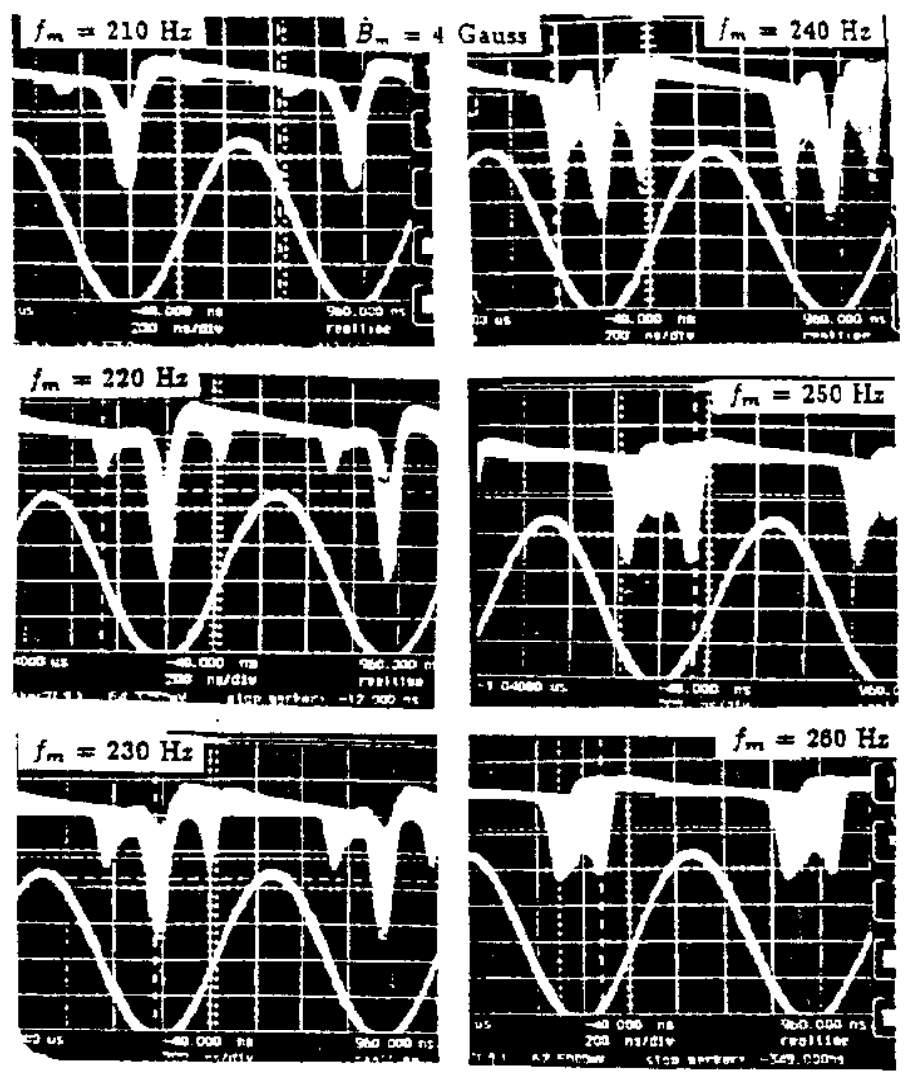

Figure 2. Longitudinal beam profile, observed from a fast sampling oscilloscope, showed the splitting of beam bunch into beamlets below the bifurcation frequency. The modulation amplitude was $\hat{B}_{m}=4$ Gauss. The sine waves in this figure are the rf waveform.

of 7 seconds, the beam-centroid displacements were measured, digitized, and recorded from signals of beam position monitors (BPM). The longitudinal phase was measured by comparing the time difference between the peak signal from a wall gap monitor and the signal from an if clock. A beam position at a high dispersion location was used to measure the momentum deviation, which is related to the off momentum closed orbit by $\Delta x_{c o}=D_{x} \frac{\Delta p}{p}$ with $D_{x} \approx 3.9 \mathrm{~m}$. A total of 8 channels were used to obtain $6 \mathrm{D}$ phase space maps with 16,384 points recorded at 10 turn intervals. Details of our detection system are reported elsewhere[2].

With a horizontal dipole (vertical field) modulation at location $s_{0}$, the horizontal closed orbit becomes[3], $x_{c o}(t)=$ $\frac{\sqrt{\beta_{x}(s) \beta_{x}\left(s_{0}\right)}}{2 \sin \pi \nu_{x}} \theta(t) \cos \left(\pi \nu_{x}-\left|\phi_{x}(s)-\phi_{x}\left(s_{0}\right)\right|\right)$, where $\theta(t)=$ $\frac{B_{m}(t) l}{B_{\rho}}=\hat{\theta} \sin \omega_{m} t$ with $\hat{\theta}=\frac{\dot{B}_{m} l}{B_{\rho}}$. Furthermore, if the dispersion function at the modulating dipole location is not zero, the path length is also modulated. The change of the circumference is given by $\Delta C=D_{x} \theta(t)$, where $D_{x}$ is the dispersion function at the modulation dipole location. The corresponding of phase difference becomes, $\Delta \phi=2 \pi h \frac{\Delta f}{C}$, where $C=86.8224 \mathrm{~m}$ is the circumference of the IUCF
Cooler Ring. In our experiment, the maximum if phase shift per turn was $\dot{\Delta \phi}=0.78 \times 10^{-5} \dot{B}_{m}$ [Gauss] radians. The longitudinal phase space coordinates, $\left(\phi, \frac{\Delta p}{p}\right)$, at the $n$th and the $(n+1)$ th revolutions are transformed according to the following mapping equations,

$$
\begin{aligned}
& \phi_{n+1}=\phi_{n}+2 \pi h \eta\left(\frac{\Delta p}{p}\right)_{n}+\Delta \phi, \\
& \left(\frac{\Delta p}{p}\right)_{n+1}=\left(\frac{\Delta p}{p}\right)_{n}+\frac{e V}{\beta^{2} E} \sin \phi_{n+1}-\lambda\left(\frac{\Delta p}{p}\right)_{n},
\end{aligned}
$$

where the fractional momentum deviation of particles, $\left(\frac{\Delta p}{p}\right)$, is the conjugate variable to the synchrotron phase angle $\phi$. and $\lambda$ is the phase space damping parameter related to electron cooling. Thus the synchrotron equation of motion, in the presence of transverse modulation, becomes,

$$
\ddot{\phi}+2 \alpha \dot{\phi}+\omega_{s}^{2} \sin \phi=\omega_{m} \omega_{s} a \cos \omega_{m} t+2 \alpha \omega_{s} a \sin \omega_{m} t,
$$

with the damping coefficient $2 \alpha=\frac{w_{0}}{2 \pi} \lambda$, and $a$ is given by,

$$
a=\frac{h \omega_{0} D_{x} \dot{\theta}}{\omega_{s} \hat{C}}=\frac{\omega_{0}}{2 \pi \omega_{s}} \dot{\Delta \phi}
$$

With electron current $0.75 \mathrm{~A}$, the damping time for the $45 \mathrm{MeV}$ protons was measured to be about $0.4 \mathrm{sec}$ or $\alpha=$ $2.5 \mathrm{~s}^{-1}$, which was indeed small compared with $\omega_{s}=$ $1646 \mathrm{~s}^{-1}$.

Even though the cooling was weak, sufficient time was allowed before making measurements that the transient solution of $\mathrm{Eq}$.(2) was damped out. Let the stationary solution of this system be given by, $\phi \approx g \sin \left(\omega_{m} t-\chi\right)$, where we have used a single harmonic approximation. Expanding the term $\sin \phi$ in Eq.(2) up to the first harmonic, we obtain the equation for the modulation amplitude $g$ as,

$$
\left[-\omega_{m}^{2} g+2 \omega_{s}^{2} J_{1}(g)\right]^{2}+\left[2 \alpha \omega_{m} g\right]^{2}=\left[\omega_{m} \omega, a\right]^{2}+[2 \alpha \omega, a]^{2}
$$

where $J_{1}$ is the Bessel function[4] of order 1. Fig. 1 shows the longitudinal phase amplitude, measured with the wall gap monitor (diamonds), as a function of sinusoidal transverse modulation frequencies for the modulation amplitude with $\hat{B}_{m}=12$ Gauss. The amplitudes of the steady state solutions of Eq.(4) are also shown in Fig.1 with $\alpha=2.5 \mathrm{~s}^{-1}$ and the parameter $a$ obtained from Eq.(3). Note here that there are multiple solutions of $\mathrm{Eq}_{\mathrm{q}}(4)$ when the modulation frequency is below the critical bifurcation frequency[5], $\omega_{c}$.

The existence of a unique phase factor $\chi$ for the solutions implies that trajectories are attracted to a single phase space point rotating with modulation frequency $\omega_{m}$. Fig. 2 gives the measured longitudinal beam profile in time domain integrated over many synchrotron periods as shown on a fast sampling digital oscilloscope, which is triggered at the of frequency. The modulation amplitude was $\dot{B}_{m}=4$ Gauss. The modulation frequencies were $210,220, \ldots, 260$ $\mathrm{Hz}$. Using a fast sampling oscilloscope for a single trace, the bunch was indeed observed to split into two bearnlets located at amplitudes corresponding to the steady state solutions of the dissipative parametric system. Both of 

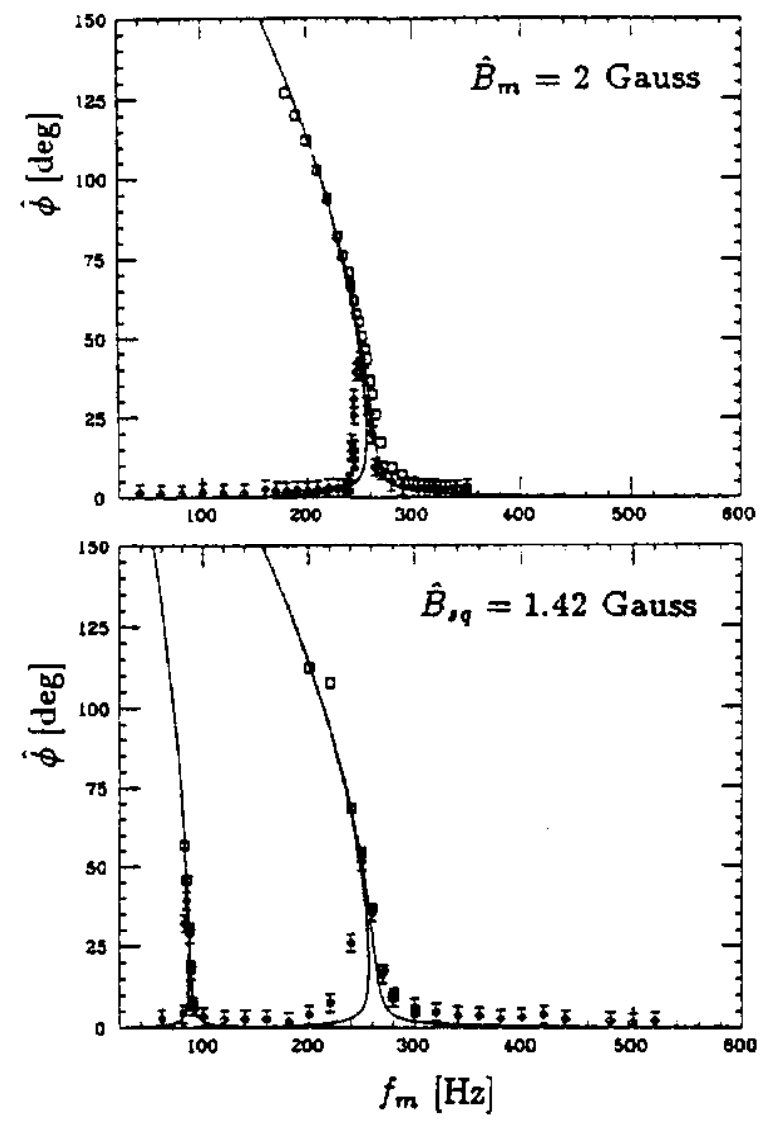

Figure 3. The phase amplitude of outer beamlet measured from a fast sampling oscilloscope and the phase amplitude measured from a wall gap monitor as functions of modulation frequency for the sinusoidal wave at $\dot{B}_{m}=2$ Gauss, and for a square wave at $\dot{B}_{m}=1.42$ Gauss. Here, $\hat{\phi}=g$.

these two beamlets rotate in the synchrotron phase space at the modulating frequency, measured from the FFT of the phase signal. As the modulating frequency is increased towards the synchrotron frequency, the outer peak moves in and its population intensity increases. When the modulating frequency was set higher than $\omega_{c}$, the center peak disappeared (see the $260 \mathrm{~Hz}$ data of Fig.2).

Fig. 3 shows the phase amplitude of the outer beamlet (squares) measured with the oscilloscope and the phase amplitude obtained from the phase detector (diamonds). Note here that our phase detector was not intended for use with more than one beam bunch present. It seems that our phase detector measured the centriod of these two beamlets shown in Figs. 1 and 3. Only when the outer beamlet becomes the dominant charge distribution, the phase detector was able to measure its phase. In the lower part of Fig. 3, the phase response data from a square wave modulation are shown. Since the square wave can be expanded in a Fourier series, $\frac{4}{\pi} \hat{B}_{s q} \sin \omega_{s q} t+\frac{4}{3 \pi} \hat{B}_{s q} \sin 3 \omega_{s q} t+\cdots$, the

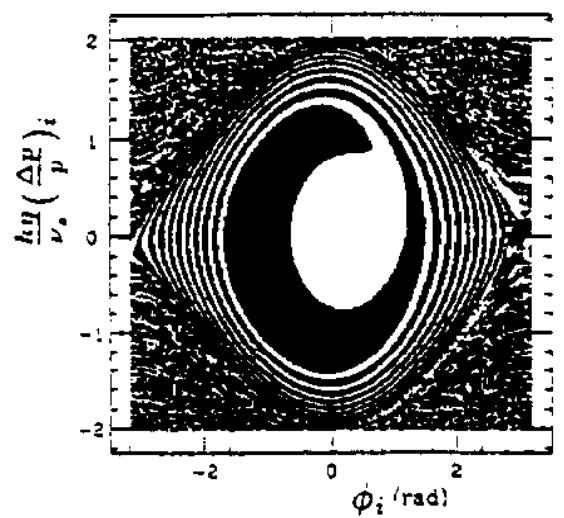

Figure 4. Initial phase space coordinates, obtained from numerical simulation of $\mathrm{Eq} .(1)$, which converge to the outer attractor are shown for $\hat{B}_{m}=4$ Gauss and $f_{m}=240 \mathrm{~Hz}$.

peak response was expected and observed at a modulation frequency of $87.3 \mathrm{~Hz}$.

A computer simulation based on Eq.(1) has been performed to demonstrate the strange attractor nature of the system. One of the results is shown in Fig. 4, where the black region corresponds to initial phase space coordinates converging toward the outer attractor. The complementary phase space coordinates converge mostly to the inner attractor except a small patch of phase space coordinates located on the boundary of the separatrix, which will converge toward two attractors located on the separatrix. The relative population of the inner and outer attractors observed from Fig. 2 can also be understood qualitatively from numerical simulations.

\section{REFERENCES}

[1] E.M. McMillan, Phys. Rev., 68, 143 (1945); V.I. Veksler, Compt. Rend. Acad. Sci. U.R.S.S., 43, 329 (1944); 44, 365 (1944).

[2] S.Y. Lee, et al., Phys. Rev. Lett. 67, 3768 (1991); D.D. Caussyn, et al., Physical Review A, 46, 7942 (1992).

[3] E.D. Courant and H.S. Snyder, Ann. Phys. $\underline{3}, 1$ (1958).

[4] M. Abramowitz and I.A. Stegun, eds, Handbook of Mathmatical Functions, National Bureau of Standard, (1975).

[5] M. Ellison, et al., Phys. Rev. Lett. 70, 591 (1993). 